\title{
Significant Risk Factors for Postoperative Enlargement of Basal Ganglia Hematoma after Frameless Stereotactic Aspiration : Antiplatelet Medication and Concomitant IVH
}

\author{
Wonsoo Son, M.D., Jaechan Park, M.D., Ph.D. \\ Department of Neurosurgery, Kyungpook National University School of Medicine, Daegu, Korea
}

Objective : Frameless stereotactic aspiration of a hematoma can be the one of the treatment options for spontaneous intracerebral hemorrhage in the basal ganglia. Postoperative hematoma enlargement, however, can be a serious complication of intracranial surgery that frequently results in severe neurological deficit and even death. Therefore, it is important to identify the risk factors of postoperative hematoma growth.

Methods : During a 13-year period, 101 patients underwent minimally invasive frameless stereotactic aspiration for basal ganglia hematoma. Patients were classified into two groups according to whether or not they had postoperative hematoma enlargement in a computed tomography scan. Baseline demographic data and several risk factors, such as hypertension, preoperative hematoma growth, antiplatelet medication, presence of concomitant intraventricular hemorrhage (IVH), were analysed via a univariate statistical study.

Results : Nine of 101 patients (8.9\%) showed hematoma enlargement after frameless stereotactic aspiration. Among the various risk factors, concomitant IVH and antiplatelet medication were found to be significantly associated with postoperative enlargement of hematomas.

Conclusion : In conclusion, our study revealed that aspirin use and concomitant IVH are factors associated with hematoma enlargement subsequent to frameless stereotactic aspiration for basal ganglia hematoma.

Key Words : Stereotactic techniques · Suction · Basal ganglia hemorrhage · Risk factors.

\section{INTRODUCTION}

Treatment options for spontaneous intracerebral hemorrhage (SICH) include conservative medicine and surgery. Although the latter is controversial, those in favor cite the following benefits : prevention of herniation, reduction of intracerebral pressure, and minimizing mass effect on the surrounding tissue $e^{11}$.

The benefits of surgical evacuation of hematomas can only be achieved with appropriate patient selection. Randomized clinical investigations have shown that in cases where evacuation may be expected to yield favorable outcomes, minimally invasive procedures, such as burr-hole stereotactic aspiration, can provide a good option, especially with deep hemato-

- Received : September 26, 2016 •Revised : December 21, 2016 •Accepted : February 28, 2017

- Address for reprints : Jaechan Park, M.D., Ph.D.

Department of Neurosurgery, Kyungpook National University School of Medicine, 130 Dongdeok-ro, Jung-gu, Daegu 41944, Korea Tel : +82-53-200-5647, Fax : +82-53-423-0504, E-mail : jparkmd@hotmail.com

This is an Open Access article distributed under the terms of the Creative Commons Attribution Non-Commercial License (http://creativecommons.org/licenses/by-nc/4.0) which permits unrestricted non-commercial use, distribution, and reproduction in any medium, provided the original work is properly cited. 
$\operatorname{mas}^{11,19)}$. An important concern with the adoption of the surgical approach is the possibility of hematoma enlargement and consequent edema and mass effect. Although rare, postoperative hematoma growth is a complication of intracranial surgery that frequently results in severe neurological deficit and even death ${ }^{2,3,8,9,13,14,17,18,20,22,25,26)}$. Therefore, it is important to identify the risk factors of postoperative hematoma growth. In this study, we attempted to identify the risk factors of postoperative hematoma enlargement at Kyungpook National University Hospital, particularly after frameless stereotactic aspiration of SICH involving the basal ganglia.

\section{MATERIALS AND METHODS}

\section{Patients}

We investigated 452 consecutive patients treated for $\mathrm{SICH}$ at Kyungpook National University Hospital over the course of 13 years between January 2002 and June 2015. Among them, 101 patients underwent minimally invasive frameless stereotactic aspiration for basal ganglia hematoma. The inclusion criteria were as follows : 1) basal ganglia hematoma, 2) treatment with frameless stereotactic aspiration, 3) no systemic bleeding diathesis, and 4) no angiographic evidence of abnormal vascular or structural causes of hemorrhage in angiographic studies, such as digital subtraction angiography, magnetic resonance angiography, or computed tomography angiography. We excluded patients with underlying vascular or hematological disorders, hematoma at sites other than the basal ganglia, or underlying hematologic disorders and those who underwent craniotomy.

\section{Data collection}

This study involved a retrospective investigation of the medical records maintained at Kyungpook National University Hospital. We collected data on baseline demographic factors and medical history, such as gender, age, and side of hematoma. To determine the risk factors of postoperative hematoma growth, other factors taken into consideration included preoperative volume of the hematoma; time interval between admission and surgery; and history of antiplatelet medication, hypertension, and intraventricular hemorrhage.

\section{Postoperative hematoma enlargement based on computed tomography scan}

Computerized tomography (CT) scans were performed using 5-mm-thick slices, and the hematoma volume was calculated using an area calculation program built into the picture archiving communication system. Postoperative hematoma enlargement was defined as an increase in the volume of intraparenchymal hemorrhage in the CT image obtained 12 hours after frameless stereotactic aspiration as compared to the preoperative hematoma (Fig . 1).

\section{Surgical procedure}

Hematoma evacuation was performed under general anesthesia. A neuronavigation system was used to determine the exact location of the hematoma for the placement of the catheter (Kolibri Cranial Navigation system; Brainlab AG, Munich, Germany). A burr hole was created using a high-speed drill, and a 10.5-Fr catheter was placed into the clot under neuronavigation guidance. Thereafter, careful manual evacuation of the hematoma was attempted using a syringe. Further, a catheter was placed to ensure complete drainage of the hematoma.

\section{Platelet transfusion regimen}

In the case of patients who had a history of using antiplatelets, such as aspirin or clopidogrel, 10 units of platelets were administered to the patient routinely to rule out the effect of antiplatelet agents. Platelet transfusion was done immediately after hospitalization. The amount of transfusion was the same in all cases.

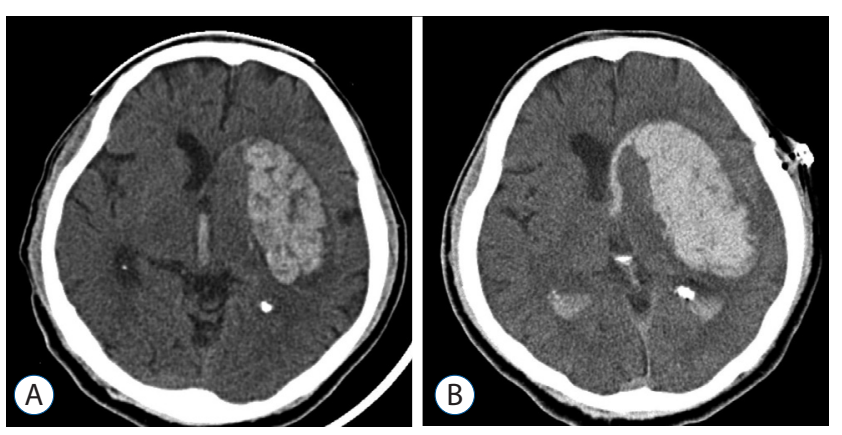

Fig. 1. Brain CT scan of patient with postoperative hematoma enlargement. Preoperative CT (A) shows basal ganglia hemorrhage with concomitant intraventricular hemorrhage. Follow-up CT 12-hours after operation (B) shows hematoma enlargement in basal ganglia in spite of stereotactic aspiration. CT : computed tomography. 


\section{Statistical analysis}

Statistical analyses were performed using a commercially available statistics software (SPSS version 19.0; SPSS, Inc., Chicago, IL, USA). Univariate analysis was carried out to determine the variables affecting postoperative hematoma enlargement risk. T-test and Fisher's exact test were used for risk factor evaluation. A standard $p=0.05$ was used to indicate a statistically significant difference between the comparison groups.

\section{RESULTS}

In all, 101 patients were enrolled in this study. Table 1 summarizes the baseline characteristics of the patients. Nine of 101 patients $(8.9 \%)$ showed hematoma enlargement after stereotactic aspiration. In order to compare the risk factors, the cases were classified into two groups according to whether they had postoperative hematoma enlargement or not : the non-postoperative hematoma enlargement group (group A), and the postoperative hematoma enlargement group (group B).

The patients included 76 men and 25 women. The mean age in groups A and B was $46.3 \pm 16.6$ and $51.4 \pm 15.5$ years, respectively. Group A included 70 men and 22 women, while group $B$ consisted of 6 men and 3 women. Of the 101 patients, 47 had hematoma on the left side, while 54 had hematoma on the right side. No intergroup differences were noted in terms of age, gender, and side of the hematoma.

Table 1. Baseline characteristics of 101 patients who underwent stereotactic aspiration of basal ganglia $\mathrm{SICH}$

\begin{tabular}{lccc}
\hline Variable & $\begin{array}{c}\text { Non-postoperative } \\
\text { hematoma growth } \\
\text { group (group A) } \\
(\mathbf{n}=\mathbf{9 2})\end{array}$ & $\begin{array}{c}\text { Postoperative } \\
\text { hematoma growth } \\
\text { group (group B) } \\
(\mathbf{n}=9)\end{array}$ & p-value \\
\hline Age (years) & $\begin{array}{c}46.3 \pm 16.6 \\
(29.7-62.9)\end{array}$ & $\begin{array}{c}51.4 \pm 15.5 \\
(35.9-66.9)\end{array}$ & $0.367^{*}$ \\
Gender & & & $0.686^{\dagger}$ \\
Male & $70(76)$ & $6(66)$ & \\
Female & $22(24)$ & $3(34)$ & \\
Left/right & $44 / 48$ & $3 / 6$ & $0.498^{\dagger}$ \\
\hline
\end{tabular}

Values are presented as mean \pm standard deviation (range) or number (\%). *Two sample t-test, ${ }^{\dagger}$ Fisher's exact test. SICH : spontaneous intracerebral hemorrhage
The differences between the two groups in terms of postoperative hematoma enlargement are shown in Table 2. The mean preoperative volume of the hematoma in group A was $46.40 \pm 16.84$, while that in group B was $49.15 \pm 13.98$. The preoperative hematoma in group B was slightly greater than that in group A, but the difference was not statistically significant ( $p=0.576)$. The time interval was defined as the time from hospital admission to the start of the surgery. The time interval in group A was longer than that in group B, although the difference had no statistical significance $(p=0.299)$. All patients in group B had hypertension, but there was no statistical correlation between postoperative hematoma enlargement and hypertension $(p=0.350)$.

CT scans showed that 19 patients from group A and 5 patients in group $B$ had intraventricular hemorrhage in addition to intracerebral hemorrhage. The percentage of accompanying intraventricular hemorrhage was significantly higher in group B (55.6\%) than in group A $(20.7 \%)(p=0.033)$. A total of seven patients, five from group $\mathrm{A}$ and two from group $\mathrm{B}$, showed enlargement of the hematoma before initiation of the stereotactic aspiration surgery. No statistical correlation was noted between preoperative and postoperative hematoma enlargement. Fifteen patients had a history of taking antiplatelet medication, namely aspirin and this factor was found to be statistically correlated with postoperative hematoma enlargement.

Table 2. Evaluation of risk factors of Hematoma growth after stereotactic aspirations of basal ganglia $\mathrm{SICH}$

\begin{tabular}{|c|c|c|c|}
\hline Variable & $\begin{array}{c}\text { Non- } \\
\text { postoperative } \\
\text { hematoma } \\
\text { growth group } \\
\text { (group } A)(n=92)\end{array}$ & $\begin{array}{l}\text { Postoperative } \\
\text { hematoma } \\
\text { growth group } \\
\text { (group B) }(n=9)\end{array}$ & $p$-value \\
\hline $\begin{array}{l}\text { Mean pre op } \\
\text { hematoma } \\
\text { volume }(\mathrm{mL})\end{array}$ & $46.29 \pm 16.56$ & $51.44 \pm 15.49$ & $0.367^{*}$ \\
\hline Time interval (min) & 1175.38 & 637.56 & $0.299 *$ \\
\hline HTN & $81(88)$ & $9(100)$ & $0.592^{\dagger}$ \\
\hline IVH & $19(20.7)$ & $5(55.6)$ & $0.033^{\dagger}$ \\
\hline $\begin{array}{l}\text { Pre op hematoma } \\
\text { growth }\end{array}$ & $5(5.4)$ & $2(22.2)$ & $0.118^{\dagger}$ \\
\hline $\begin{array}{l}\text { Antiplatelet } \\
\text { medication }\end{array}$ & $10(10.9)$ & $5(55.6)$ & $0.003^{\dagger}$ \\
\hline
\end{tabular}

Values are presented as mean \pm standard deviation or number (\%). ${ }^{*}$ Two sample t-test, ${ }^{\dagger}$ Fisher's exact test. $\mathrm{SICH}$ : spontaneous intracerebral hemorrhage, HTN : hypertension, IVH : intraventricular hemorrhage 
Thus, among the various risk factors, intraventricular hemorrhage and antiplatelet medication were found to be significantly associated with postoperative enlargement of hematoma.

\section{DISCUSSION}

We analyzed the risk factors expected to affect postoperative hematoma enlargement, including hypertension, presence of intraventricular hemorrhage, volume of preoperative hematoma, time interval between admission and surgery, and antiplatelet medication. Statistical analysis involving all these factors showed that most of the abovementioned factors were not correlated with postoperative hematoma enlargement. However, two risk factors, a history of antiplatelet medication and preoperative intraventricular hemorrhage (IVH), were statistically correlated to postoperative hematoma enlargement.

Although we speculated that preoperative hematoma enlargement may strongly influence postoperative hematoma enlargement, our results failed to demonstrate a statistically significant association. In agreement with our results, $\mathrm{Xu}$ et al. $^{25)}$ reported that preoperative hematoma enlargement does not raise the possibility for increased risk of rebleeding after aspiration of spontaneous intracerebral hemorrhage. Investigators have identified several risk factors of hematoma enlargement in intracerebral hemorrhage, including time after onset, irregularity of hematoma shape, blood pressure, and antiplatelet medication. Fujii et al. ${ }^{8)}$ previously reported that the risk of hematoma enlargement was particularly high in patients who were admitted shortly after onset, those who were heavy alcohol drinkers, those with irregularly shaped hematomas, and those with disturbed consciousness.

\section{Antiplatelet medication}

A noteworthy finding of this study is that antiplatelet medication was significantly associated with the postoperative enlargement of hematomas. Previous studies have shown that antiplatelet medication is linked to increases in the size of the clot and SICH volume ${ }^{16,21)}$ as well as poor prognosis ${ }^{24)}$. However, no study to date has shown a correlation between antiplatelet medication and postoperative hematoma enlargement. Improvement in platelet activity through platelet transfusion has been recommended as a feasible option to reduce the possibil- ity of hematoma enlargement and improve outcomes ${ }^{21)}$. At Kyungpook National University Hospital, 10 platelet units are administered to SICH patients known to be taking antiplatelet medications to counter the effect of the medication. Platelet transfusion was administered immediately after hospitalization. The amount of transfusion was the same in all the cases. Possible explanation for the increased risk of postoperative hematoma enlargement in patients taking antiplatelet agents despite platelet transfusion is that the amount of platelets may be inadequate to counter the effect of antiplatelet agents. However, in our study, we could not identify the effect of platelet transfusion. By a more quantitative test, it would be necessary to determine the effectiveness of platelet transfusion. Engel-Haber et al. ${ }^{6)}$ demonstrated that platelet transfusion should be provided only to those most likely to benefit from this treatment. Further, they attempted to evaluate the effect of aspirin using quantitative tests such as the aspirin response test (VerifyNow aspirin assay; Accriva Diagnostics, San Diego, CA, USA) ${ }^{21)}$, which measures the extent of the action of aspirin on its target, cycloocygenase-1. A serum aspirin response unit level of $<550$ is indicative of reduced platelet activity and sufficient aspirin functionality. The sensitivity and specificity of the test for platelet inhibition induced by aspirin was shown to be $100 \%$ and $96 \%$, respectively ${ }^{6}$. Engel-Haber et al. ${ }^{6)}$ showed that platelet transfusion after SICH does not reduce the expansion rates of hematoma in aspirin responders. However, their study had some limitations, including a lack of a specific transfusion protocol and specific data regarding the amount of platelet administered. Therefore, it may be difficult to accurately analyze the relevance of the quantity of platelet transfusion and hematoma enlargement.

Monitoring platelet function would facilitate the evaluation of exposure to antiplatelet medications and guide hemostatic interventions; however, further investigations are necessary in this regard ${ }^{11)}$. To our knowledge, only a few reports have been published on the quantitative analysis of the effect of platelet transfusion, using tests such as the aspirin response test and platelet function assays ${ }^{21,23)}$. We hypothesize that the adequacy of platelet transfusion could be estimated by using quantitative tests. Further, the data obtained via these tests would be helpful to lower the risk of postoperative hematoma enlargement.

Although the effects of a platelet transfusion on the survival of SICH patients taking antiplatelet agents has been discussed by several authors, there still remains some ambiguity regard- 
Risk Factors for Postoperative Enlargement of Basal Ganglia Hematoma I Son W, et al.

ing the effect of platelet transfusion on the outcome of $\mathrm{SICH}^{1,4,6,12)}$. Therefore, large prospective studies are necessary to identify the risk factors for postoperative hematoma enlargement and the value of platelet transfusion in such cases.

\section{Concomitant IVH}

Another risk factor of postoperative hematoma enlargement found in this study was concomitant IVH. IVH can originate and remain confined to the ventricular system (primary IVH, $30 \%$ of cases) or occur as a result of an intraparenchymal or subarachnoid hemorrhage into the ventricular system (secondary IVH, 70\% of cases). Secondary IVH is commonly caused by hypertensive spontaneous SICH, ruptured cerebral aneurysm, and arteriovenous malformation ${ }^{77}$.

Several studies have shown that IVH is a significant predictive factor of poor outcome and mortality ${ }^{5,15)}$. Important causes for poor outcome and mortality associated with edema are inflammation and concomitant hydrocephalus caused by $\mathrm{IVH}^{10)}$. Several papers have highlighted the importance of IVH in predicting the outcome and development of hydrocephalus $^{11)}$. Hallevi et al. ${ }^{10)}$ studied the anatomic relationship between SICH and IVH and found that IVH occurs in nearly half the patients with spontaneous SICH and that IVH is related to the volume and location of SICH. IVH tended to commonly occur when SICH occurred in the thalamus, putamen, or lobar region. SICH occurring deep in the brain has been found to extend into the ventricular spaces, leading to IVH.

Although many studies have shown that IVH is an important risk factor of poor outcome, the significance of IVH on postoperative growth of hematomas has not been investigated hitherto. Our study demonstrated a significant association between concomitant IVH and postoperative hematoma growth. Interestingly, IVH is not only a predictor of concomitant hydrocephalus, but also a risk factor of postoperative hematoma enlargement.

We attempted to figure out why postoperative hematoma enlargement is related to IVH. IVH associated with basal ganglia ICH unlikely originates from the ventricular system. In most cases, hemorrhage in the ventricular system originates from extended ICH in the basal ganglia. In other words, ICH extended into a ventricle has a tendency to expand. Sustained arterial bleeding may be the cause of hematoma extension. Accordingly, we can hypothesize that concomitant IVH has significant implications related to the tendency for hematoma extension. In addition, hematoma extension can be maintained even after aspiration surgery. Hence, SICH aspiration in patients with IVH should be performed with extreme caution.

Some of the limitations of this study are its retrospective nature and small study population from a single center. More large-scale studies covering multiple centers are necessary to confirm our findings.

\section{CONCLUSION}

In conclusion, our study revealed that aspirin use and concomitant IVH are factors associated with enlargement of hematoma subsequent to frameless stereotactic aspiration for basal ganglia hematoma. In this study, platelet transfusion in patients receiving antiplatelet agents was not significant in suppressing the increase in SICH after aspiration surgery, further investigations are necessary to confirm the value of platelet transfusion in the management of postoperative hematoma enlargement. Also, close quantitative monitoring is necessary to ensure safety the value of platelet transfusion. Furthermore, we were able to identify IVH as an additional risk factor that influences postoperative hematoma growth.

\section{References}

1. Baharoglu MI, Cordonnier C, Al-Shahi Salman R, de Gans K, Koopman MM, Brand $A$, et al. : Platelet transfusion versus standard care after acute stroke due to spontaneous cerebral haemorrhage associated with antiplatelet therapy (Patch): a randomised, open-label, rhase 3 trial. Lancet 387 : 2605-2613, 2016

2. Broderick JP, Diringer MN, Hill MD, Brun NC, Mayer SA, Steiner T, et al. : Determinants of intracerebral hemorrhage growth: an exploratory analysis. Stroke 38 : 1072-1075, 2007

3. Brott T, Broderick J, Kothari R, Barsan W, Tomsick T, Sauerbeck L, et al. : Early hemorrhage growth in patients with intracerebral hemorrhage. Stroke 28 : 1-5, 1997

4. Creutzfeldt CJ, Weinstein JR, Longstreth WT Jr., Becker KJ, McPharlin TO, Tirschwell DL : Prior antiplatelet therapy, platelet infusion therapy, and outcome after intracerebral hemorrhage. J Stroke Cerebrovasc Dis $18: 221-228,2009$

5. el Chami B, Milan C, Giroud M, Sautreaux JL, Faivre J : Intracerebral hemorrhage survival: French register data. Neurol Res 22 : 791-796, 2000 
6. Engel-Haber E, Horev A, Chablani P, Bornstein NM, Jadhav A, Jovin TG, et al. : Aspirin response test role in platelet transfusion following intracerebral hemorrhage. Clin Neurol Neurosurg 137 : 12-14, 2015

7. Engelhard HH, Andrews CO, Slavin KV, Charbel FT : Current management of intraventricular hemorrhage. Surg Neurol 60 : 15-21; discussion 21-22, 2003

8. Fujii Y, Takeuchi S, Sasaki O, Minakawa T, Tanaka R : Multivariate analysis of predictors of hematoma enlargement in spontaneous intracerebral hemorrhage. Stroke 29 : 1160-1166, 1998

9. Fujii Y, Tanaka R, Takeuchi S, Koike T, Minakawa T, Sasaki 0 : Hematoma enlargement in spontaneous intracerebral hemorrhage. J Neurosurg $80: 51-57,1994$

10. Hallevi H, Albright KC, Aronowski J, Barreto AD, Martin-Schild S, Khaja $A M$, et al. : Intraventricular hemorrhage: anatomic relationships and clinical implications. Neurology $70: 848-852,2008$

11. Hemphill JC 3rd, Greenberg SM, Anderson CS, Becker K, Bendok BR, Cushman $M$, et al. : Guidelines for the management of spontaneous intracerebral hemorrhage: a guideline for healthcare professionals from the American Heart Association/American Stroke Association. Stroke 46 : 2032-2060, 2015

12. Ishibashi A, Yokokura $Y$, Adachi $H$ : Is antiplatelet therapy for the prevention of ischemic stroke associated with the prognosis of intracerebral hemorrhage? Kurume Med J 55 : 71-75, 2008

13. Juvela $S$ : Risk factors for impaired outcome after spontaneous intracerebral hemorrhage. Arch Neurol 52 : 1193-1200, 1995

14. Kaneko M, Tanaka K, Shimada T, Sato K, Uemura K : Long-term evaluation of ultra-early operation for hypertensive intracerebral hemorrhage in 100 cases. J Neurosurg $58: 838-842,1983$

15. Karnik R, Valentin A, Ammerer HP, Hochfelner A, Donath P, Slany J : Outcome in patients with intracerebral hemorrhage: predictors of survival. Wien Klin Wochenschr 112 : 169-173, 2000

16. Kawamata T, Takeshita M, Kubo O, Izawa M, Kagawa M, Takakura K : Management of intracranial hemorrhage associated with anticoagulant therapy. Surg Neurol 44 : 438-442; discussion 443, 1995

17. Mendelow AD, Gregson BA, Fernandes HM, Murray GD, Teasdale GM,
Hope DT, et al. : Early surgery versus initial conservative treatment in patients with spontaneous supratentorial intracerebral haematomas in the international surgical trial in intracerebral haemorrhage (STICH): a randomised trial. Lancet 365 : 387-397, 2005

18. Mendelow AD, Gregson BA, Rowan EN, Murray GD, Gholkar A, Mitchell PM; STICH II Investigators : Early surgery versus initial conservative treatment in patients with spontaneous supratentorial lobar intracerebral haematomas (STICH II): a randomised trial. Lancet 382 : 397-408, 2013

19. Montes JM, Wong JH, Fayad PB, Awad IA : Stereotactic computed tomographic-guided aspiration and thrombolysis of intracerebral hematoma : protocol and preliminary experience. Stroke $31: 834-840,2000$

20. Morgenstern LB, Demchuk AM, Kim DH, Frankowski RF, Grotta JC : Rebleeding leads to poor outcome in ultra-early craniotomy for intracerebral hemorrhage. Neurology 56 : 1294-1299, 2001

21. Naidech AM, Jovanovic B, Liebling S, Garg RK, Bassin SL, Bendok BR, et al. : Reduced platelet activity is associated with early clot growth and worse 3-month outcome after intracerebral hemorrhage. Stroke 40 : 2398-2401, 2009

22. Palmer JD, Sparrow OC, lannotti F : Postoperative hematoma: a 5-year survey and identification of avoidable risk factors. Neurosurgery 35 : 1061-1064; discussion 1064-1065, 1994

23. Rand ML, Leung R, Packham MA : Platelet function assays. Transfus Apher Sci 28 : 307-317, 2003

24. Suzuki Y, Kitahara T, Soma K, Konno S, Sato K, Suzuki S, et al. : Impact of platelet transfusion on survival of patients with intracerebral hemorrhage after administration of anti-platelet agents at a tertiary emergency center. PLoS One 9 : e97328, 2014

25. Xu F, Tang Z, Luo X, Kang H, Hu Q, Wang W, et al. : No evidence of preoperative hematoma growth representing an increased postoperative rebleeding risk for minimally invasive aspiration and thrombolysis of ICH. Br J Neurosurg 24 : 268-274, 2010

26. Zuccarello M, Brott $T$, Derex $L$, Kothari R, Sauerbeck L, Tew J, et al. : Early surgical treatment for supratentorial intracerebral hemorrhage: a randomized feasibility study. Stroke 30 : 1833-1839, 1999 